\title{
Dependence of the self-diffusion coefficient on the sorbate concentration: A two-dimensional lattice gas model with and without confinement
}

\author{
Shreyas Y. Bhide \\ Solid State and Structural Chemistry Unit, Indian Institute of Science, Bangalore-560012, India \\ S. Yashonath ${ }^{\text {a) }}$ \\ Solid State and Structural Chemistry Unit, Supercomputer Education and Research Centre, \\ Indian Institute of Science, Bangalore-560012, India
}

\begin{abstract}
Previous pulsed-field gradient (PFG) nuclear magnetic resonance (NMR) measurements on different sorbate-zeolite systems suggest that there exist at least five different types of dependence of self-diffusivity of the sorbate, $D$, on the concentration, $c$, of the sorbate. Sorbate-zeolite systems have been modeled as a two-dimensional lattice gas and studied by carrying out Monte Carlo simulations under different conditions. Among the different factors that have been varied are the arrangement of adsorption sites, hop length, nature and strength of the sorbate-sorbate interaction, and the degree of confinement. Surprisingly, even the simple 2-D lattice gas model could yield more than one type of $D$ vs $c$ dependence. The present study provides insight into the possible reasons for a given type of $D$ vs $c$ dependence.
\end{abstract}

\section{INTRODUCTION}

Diffusion in porous systems has been extensively studied recently. ${ }^{1-12}$ The extremely rich variety in the equilibrium and dynamical properties exhibited by these systems makes them interesting. Such a variety arises from the fact that there are a number of factors that determine their properties. ${ }^{13}$ Moreover, the fact that sorbates in porous solids are similar to guest-host complexes makes their study of wider applicability and significance. It is increasingly realized that one cannot expect diffusion in these systems to be like that in the bulk. Studies mainly on zeolites, but some on aluminophosphates as well, have revealed new phenomena that result from this "confinement." For example, these systems have been found to exhibit shut-outs, ${ }^{14}$ single-file diffusion, ${ }^{15}$ and the levitation effect. ${ }^{16}$

The dependence of the self-diffusion coefficient $D$ on the sorbate concentration $c$ is of particular importance. This has been investigated by the technique of PFG-NMR by Kärger and Pfeifer. ${ }^{17}$ Different sorbate-zeolite systems exhibit different dependences, but all of these generally fall into five different types shown in Fig. 1. For example, the selfdiffusion coefficients of small $n$-alkanes in zeolites $\mathrm{NaX}$ and silicalite decrease with increasing concentration (type I behavior). On the other hand, at low sorbate concentrations, water and ammonia in zeolite $\mathrm{NaX}$ exhibit an initial increase in the self-diffusivity $(D)$ which, at higher concentrations, saturates to a particular value (type III behavior).

It is important to understand the factors that give rise to different dependences of $D$ on $c$ observed in different guestzeolite systems. These guest-zeolite systems are rather com-

\footnotetext{
a) Also at Condensed Matter Theory Unit, Jawaharlal Nehru Center for Advanced Scientific Research.

Electronic mail: yashonat@sscu.iisc.ernet.in
}

plicated and several factors influence the self-diffusivity. The $\mathrm{Si} / \mathrm{Al}$ ratio, the nature of extra framework cations, presence of water in the zeolite framework, and the pore size distribution of the zeolitic host are some of the factors that influence the self-diffusivity. The nature of the guest molecule and the nature of its interaction with the zeolite host also influence the self-diffusivity. For example, polar molecules such as water, ammonia, etc. exhibit an increase in the selfdiffusivity with sorbate concentration, at least at low concentrations. Such a variety of factors affecting diffusivity is what makes it so difficult to obtain the influence of any given factor on the diffusivity in a laboratory experiment since, more often than not, when one of these factors is varied, the others are automatically altered. Theoretical approaches can at best handle very simple systems and are unable to handle the complexity inherent to the sorbate-zeolite systems.

Computational approaches provide a viable alternative. The numerical methods employed provide us with freedom to either include only the bare essential details or take into consideration all the complexities involved in the real system. The former would offer a better alternative whenever it is necessary to pinpoint the cause-effect relationship without ambiguity, since it would lack needless details. Further, when the complex guest-zeolite systems are replaced by appropriate model systems, these studies are greatly simplified, making it possible to carry out long simulations within reasonable computational resources often yielding as much insight as a full-scale simulation. We have, therefore, chosen to model the sorbate-zeolite systems as a two-dimensional lattice gas model.

Several workers ${ }^{18-28}$ have investigated the diffusion of atoms or molecules on a surface or inside a zeolite by Monte Carlo (MC) methods within the framework of lattice gas 

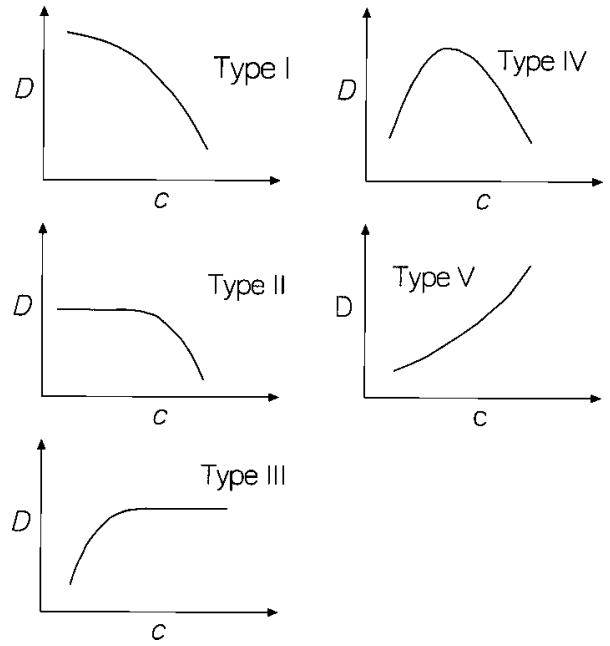

FIG. 1. Different types of dependence observed by Kärger and Pfeifer (Ref. 17) in the PFG-NMR measurements of intracrystalline self-diffusion coefficienti, $D$, on sorbate concentration, $c$.

models. A few of these studies ${ }^{18,19,28}$ employ the Metropolis Monte Carlo method to calculate the self-diffusion coefficients. Others ${ }^{22-27}$ have employed the kinetic Monte Carlo method to study diffusivity within the framework of lattice gas models. Whenever a certain physical quantity related to the system of interest is available, either from experiment or other sources, it can then be used to simulate the properties of the system realistically via the kinetic Monte Carlo method. For example, from a knowledge of the hopping rates between different adsorption sites of sorbates in zeolitic cavities, it is possible to compute the self-diffusion coefficient. Here, each Monte Carlo step is associated with a time increment. ${ }^{29}$ Kang and Weinberg, ${ }^{30,31}$ as well as Fichthorn and Weinberg, ${ }^{29}$ have investigated the three different approaches available, namely, Metropolis MC, Kawasaki dynamics, and kinetic MC on diffusion of adsorbed species within the lattice gas model. These are characterized by three different transition probabilities: $w_{m}, w_{k}$, and $w_{e}$, respectively. They conclude that when the system has reached equilibrium, a linear relationship holds between the time scales of the three methods. They further find in their investigations of the self-diffusivity that the mean-square displacement scales linearly with time and is independent of the type of the transition probability used in the simulation. The actual magnitudes of the self-diffusivities are expected to be different.

In the present study, the sorbate-zeolite systems for which PFG-NMR results are available are diverse in nature, and the hopping frequencies between different adsorption sites are not known for all the sorbate-zeolite systems. In view of this, as well as in the light of the findings of Fichthorn and Weinberg ${ }^{29}$ cited above, there is no particular advantage in using the kinetic Monte Carlo method for the present simulation. Note that in an equilibrated system Monte Carlo steps are proportional to time, ${ }^{30}$ though the proportionality constant is unknown. Since, in the present study, we have calculated self-diffusivity only after sufficient equilibration of the system has been performed, the number of Monte Carlo steps is expected to be proportional to time.
Further, since in a lattice gas model the unit of length is arbitrary, one does not attach any importance to the unit of time. Our aim here is not to reproduce the experimentally observed self-diffusivities in sorbate-zeolite systems. Instead, we are interested here in obtaining qualitative trends in self-diffusivities as a function of sorbate concentration.

We have varied in the lattice gas model the nature and arrangement of adsorption sites, the magnitude of the on-site energies, the nature and strength of the interaction amongst the sorbates, the hop length, and degree of confinement. In this study, we would like to raise the following questions: what are the factors that influence the type of dependence of the self-diffusion coefficient, $D$, on the sorbate concentration, $c$ ? What is the effect of the variation of the pattern of adsorption sites on $D$ ? What is the effect of confinement on the $D$ vs $c$ pattern? Are the different types of $D$ vs $c$ dependences obtained by Kärger and co-workers distinct? In other words, can a given sorbate-zeolite system exhibit more than one of the five types of $D$ vs $c$ dependence? We will try to answer these and related questions here.

\section{LATTICE GAS MODEL}

The model consists of a two-dimensional lattice of $50 \times 50$ sites. Of the 2500 sites, some were designated " adsorption sites" with an energy of $\epsilon_{a}$ and others were the "normal sites" with energy $\epsilon_{n}$. Note that the on-site energies for the adsorption sites are lower than the normal sites, i.e., $\left|\epsilon_{a}\right|>\left|\epsilon_{n}\right|$.

Several patterns of adsorption sites were employed with a view to understand its influence on the $D$ vs $c$ dependence. Three different spatial distributions of adsorption sites have been considered here. Figure 2 displays the patterns of adsorption sites of a $10 \times 10$ unit. These are termed as (a) plain, (b) square, (c) clustered, and (d) chessboard. The lattice was constructed by repeating the $10 \times 10$ unit in the two $(x$ - and $y$-) directions. The ratio of the number of adsorption sites to that of the total number of sites is defined as $\eta_{\text {ads }}$.

Calculations have been carried out at different concentrations, $c$, where $c$ is the number of sorbates. Double occupation of a site is forbidden, which leads to a decrease in diffusivity with $c$ whenever the number of sorbates exceeds 1250 , that is, $50 \%$ of the total number of available sites. Therefore, no calculations for concentrations higher than $50 \%$ occupancy are reported in the present study.

Nearest-neighbor (nn) sites are defined as the lattice sites nearest to a given site along the $x$ - or the $y$-direction. Only interactions between particles on nearest neighbor $(\mathrm{nn})$ sites have been considered. The nature and magnitude of nearestneighbor interactions, $\epsilon_{s s}$, can be of two types: (i) repulsive $\left(\epsilon_{s s}>0\right)$, or (ii) attractive $\left(\epsilon_{s s}<0\right)$. These may be interpreted as corresponding to two sizes, $\sigma$, of sorbates: (1) $\sigma$ $>d$ when $\epsilon_{s s}>0$, and (2) $\sigma<d$ when $\epsilon_{s s}<0$. Here, $d$ is the separation between two sorbates. The value for $\epsilon_{s s}$ in the former case is chosen large enough so that nearest-neighbor occupation is very rare. The justification for these choices of $\mathrm{nn}$ interactions arises from experimental data. The site-to-site distance, for example, in zeolite $\mathrm{NaY}^{32}$ is around $d=6 \AA$, and when a sorbate such as xenon is adsorbed, the situation corresponds to the latter case as $\sigma_{s s}=\sigma_{\mathrm{Xe}}<d$. Here, $d$, the 


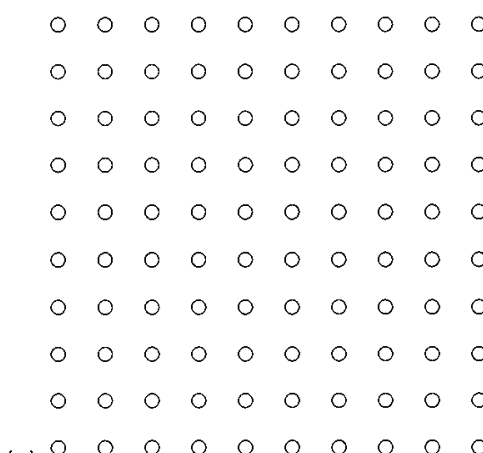

(a)

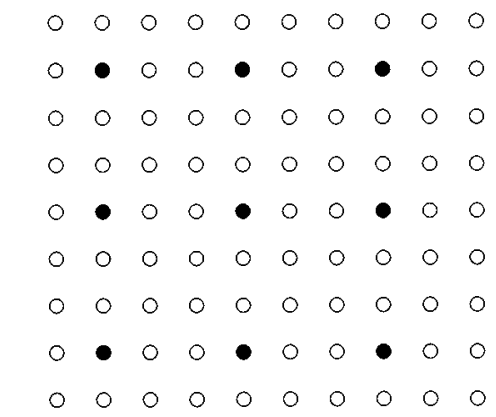

(b) 000000000000

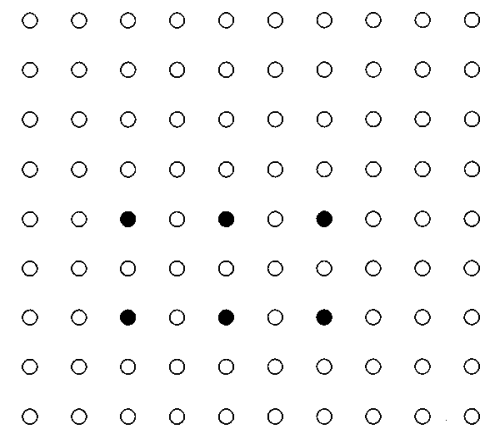

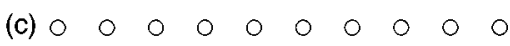

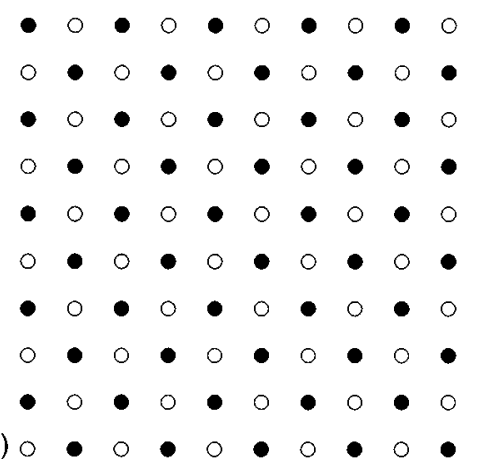

FIG. 2. Four different arrangements of adsorption sites on the basic $10 \times 10$ building unit of the lattice are shown: (a) plain, (b) square, (c) clustered, (d) chessboard. Open circles represent normal sites and filled circles represent adsorption sites. site-to-site distance lies in the attractive part $\left(\epsilon_{s s}<0\right)$ of the (6-12) Lennard-Jones potential energy curve. This situation is depicted in Fig. 3(a). On the other hand, if a large-sized sorbate such as, for example, neopentane ${ }^{33}$ is adsorbed, then $d$ lies in the region where repulsion is predominant as shown in Fig. 3(b). Another example for the case $\epsilon_{s s}>0$ is that of methane in silicalite. Molecular dynamics (MD) studies by Goodbody et al. $^{34}$ of methane in silicalite indicate that of the four available potential minima along a straight channel, only two can be occupied at any time owing to the strong repulsive interaction between methane molecules occupying two adjacent minima.

Hopping to $\mathrm{nn}$ sites as well as to next-nearest neighbor (nnn) sites is permitted. The former is referred to as single hop, denoted by $h=1$, and the latter is referred to as double hop, indicated by $h=2$. Both hops are along $x$ - or $y$-directions, and no hop along a diagonal is allowed. During double hops $(h=2)$, first an attempt is made to hop to one of the four nnn sites chosen randomly. This could be unsuccessful (i) either if the chosen nnn site is occupied (double occupation is forbidden), or (ii) if the intermediate nn site is either occupied or is an adsorption site having a lower on-site energy. In case it is unsuccessful, a hop to the intermediate nn site is attempted. This model for hop length is similar to that first employed by Barrer and $\mathrm{Jost}^{35}$ for zeolites. More recently, Azzouz et al. $^{36}$ have found that long hops are significant for particles adsorbed on surfaces. In the case of a single hop $(h=1)$, an attempt is made to hop to one of the four nn sites chosen randomly.

Confinement is introduced by restricting migration of particles from one $10 \times 10$ block to another via fewer than ten sites along the edge of the block. In the normal case where there is no confinement, migration from one block to another can take place from any site along the edge of the block. Figures 4(a) and 4(b) depict two lattices with different degrees of confinement used in this study: 6- and 1-site windows. All calculations that include confinement have been carried out with clustered arrangement of adsorption sites [see Fig. 2(c)].

\section{COMPUTATIONAL DETAILS}

All simulations have been carried out on a $50 \times 50$ lattice. The ratio $\eta_{\text {ads }}$ for the three patterns shown in Figs. 2(b),

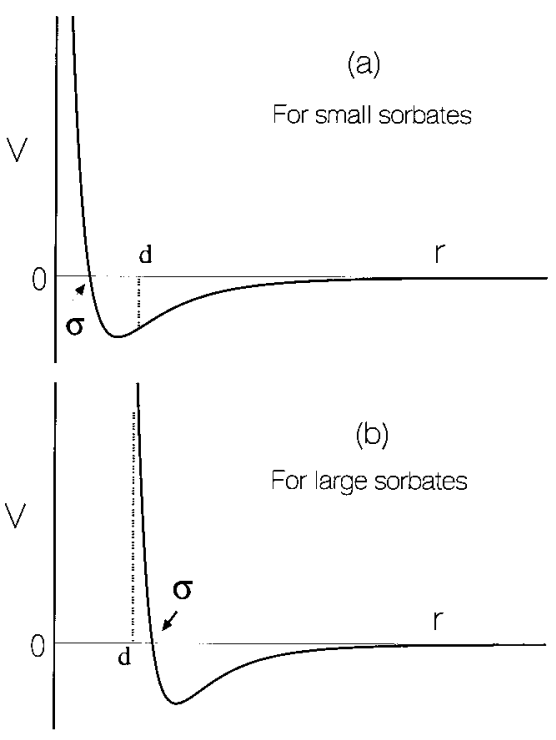

FIG. 3. Two situations where the nn interaction energies are (a) attractive $\left(\epsilon_{s s}<0\right)$ and (b) repulsive $\left(\epsilon_{s s}>0\right)$. The former corresponds to a situation where the adsorbate diameter, in Lennard-Jones interaction potential, is smaller than the nn distance. The latter corresponds to the case when the adsorbate L-J diameter is larger than the nn distance. 


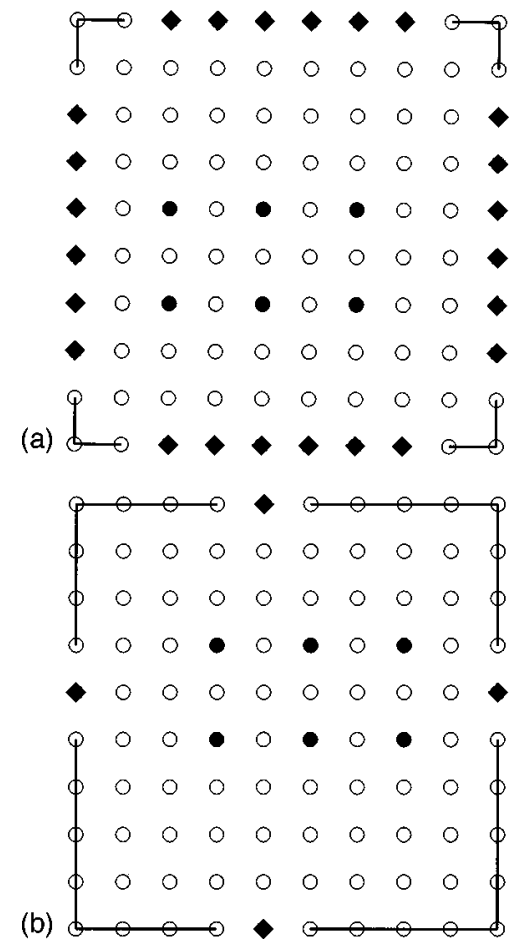

FIG. 4. Basic building units in the presence of confinement for (a) the case of a 6-site window, and (b) 1-site window are shown. All calculations with confinement have been performed on clustered arrangement of adsorption sites. Open circles represent normal sites and filled circles represent adsorption sites. Filled diamonds represent window sites through which the sorbates can leave the $10 \times 10$ block of sites.

2(c), and 2(d) are 0.1024(square), 0.06(clustered), and 0.5 (chessboard), respectively. On-site energies have been taken to be $\epsilon_{n}=-10 \mathrm{~kJ} / \mathrm{mol}$ and $\epsilon_{a}=-20 \mathrm{~kJ} / \mathrm{mol}$, in all cases.

All simulations have been carried out in the canonical (NVT) ensemble using the standard Metropolis ${ }^{37}$ Monte Carlo importance sampling algorithm employing periodic boundary conditions. In the lattice gas model, the volume $V$ is replaced by the total number of sites on the lattice. Each Monte Carlo step consists of an attempt to move each of the particles once by choosing them cyclically, in one of the four directions, chosen randomly. Probability for the hop is calculated taking into account the on-site and sorbate-sorbate interaction energies at the new and the old sites. The usual Metropolis algorithm is followed to decide if the hop is successful. Where confinement has been introduced, the number of directions to which the particle can hop to is appropriately reduced for particles near boundary.

The interaction $\epsilon_{s s}$ between the particles located on $\mathrm{nn}$ sites is taken to be either (i) $-0.5 \mathrm{~kJ} / \mathrm{mol}$ or (ii) $+20.0 \mathrm{~kJ} /$ mol. In some cases an energy of $-10 \mathrm{~kJ} / \mathrm{mol}$ has been used. The concentration of the total number of adsorbates was in the range 100 to 1250 . The value of 1250 for $c$ suggests $50 \%$ occupancy of the lattice sites. Note that a value beyond 50\% occupancy would lead to a decrease in the value of $D$ on a two-dimensional square lattice.

Each run with an equilibration phase consisting of 3000 MC steps and a production phase of $60000 \mathrm{MC}$ steps was

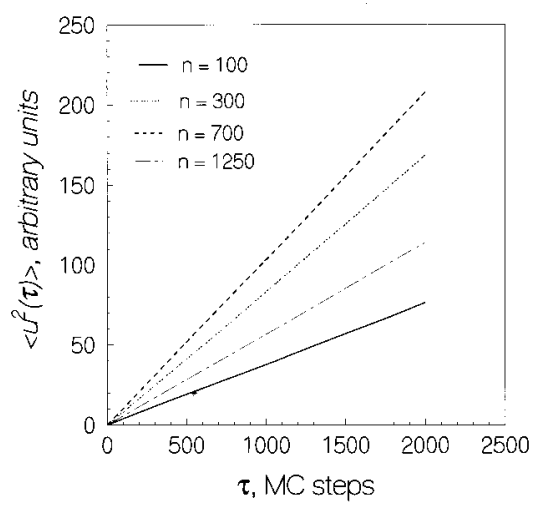

FIG. 5. Mean-square displacement, $\mathrm{u}^{2}(\tau)$ as a function of MC steps, calculated from the Monte Carlo simulation at different values of the sorbate concentration.

carried out with periodic boundary conditions. The temperature in all cases was $300 \mathrm{~K}$.

In order to obtain qualitative trends, we have calculated the self-diffusion coefficient for the two-dimensional lattice gas model from

$$
D=\frac{\left\langle\left(r\left(t_{0}+\tau\right)-r\left(t_{0}\right)\right)^{2}\right\rangle}{4 \tau}=\frac{\left\langle u^{2}(\tau)\right\rangle}{4 \tau},
$$

where $r\left(t_{0}\right)$ and $r\left(t_{0}+\tau\right)$ are two positions, separated by $\tau$ MC steps. The $\langle\ldots\rangle$ implies that averaging has been done over a number of time origins, $t_{0}$, as well as over all the particles. Typical plots of the mean-squared displacement as a function of time (MC steps), $u^{2}(\tau)$ are shown in Fig. 5 for square arrangement of adsorption sites at different sorbate concentrations for $h=2$. The linear relationship between $u^{2}(\tau)$ and the MC steps, $\tau$, seen in Fig. 5 confirms that the system has indeed reached equilibrium. The definition of the unit of time is arbitrary. We take one MC step as representing the unit of time. ${ }^{30}$ In each MC step, each of the particles present on the lattice attempts a displacement once. MC configurations were stored every tenth MC step. The meansquare displacement curve was computed from these stored steps. The estimated error in the calculated self-diffusion coefficient is found to be about $7 \%$.

\section{RESULTS AND DISCUSSION}

The principal quantity of interest in this study is the self-diffusivity. The aim of these calculations is to obtain the nature of dependence of the self-diffusivity $D$ on the sorbate concentration, $c$. The different factors that could influence the pattern of $D$ vs $c$ curves are (i) the pattern of the adsorption sites: plain, square, clustered, and chessboard pattern, (ii) interparticle interaction strength, $\epsilon_{s s}$, (iii) hop length, $h$ (single or double hops), and (iv) the effect of confinement. We would like to point out that the confinement introduced here within the lattice gas model differs from that present in a real porous solid such as zeolite. For example, in a zeolite, there would be attraction between the sorbate and the internal surface of the pore in which the sorbate is residing. Confinement introduced by us here gives rise to only geometric confinement. 


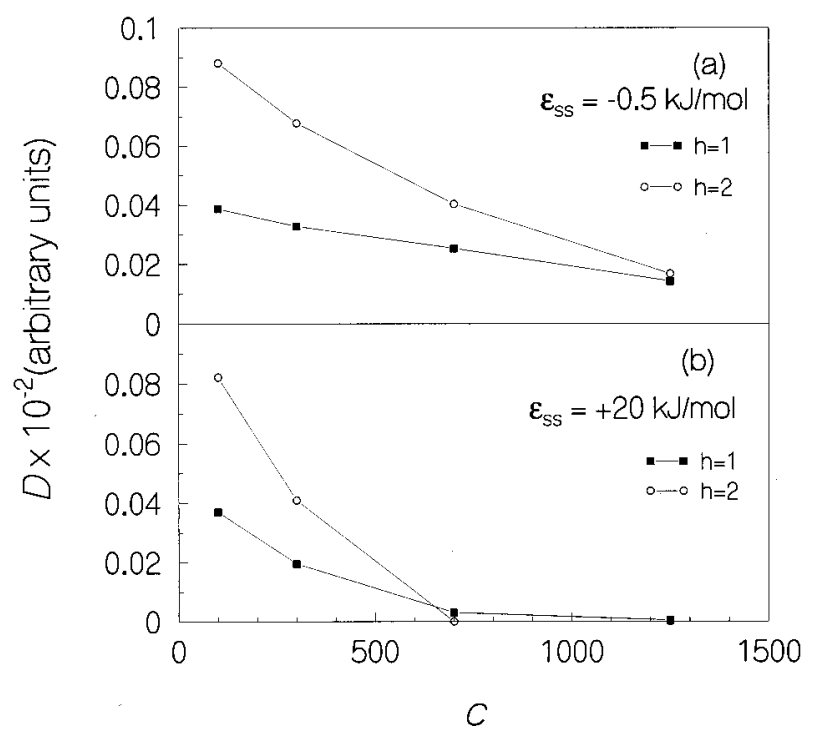

FIG. 6. Results for lattice with no adsorption sites [Fig. 2(a)]. Dependence of self-diffusivity $D$ on sorbate concentration $c$ for (a) $\epsilon_{s s}=-0.5 \mathrm{~kJ} / \mathrm{mol}$ with $h=1$ and 2. (b) $\epsilon_{s s}=+20 \mathrm{~kJ} / \mathrm{mol}$ with $h=1$ and 2 .

We have varied the above factors and obtained $D$ vs $c$ curves under different conditions. There could be several other factors such as temperature, etc. which are also expected to influence the dependence of $D$ on $c$, but in this investigation we restrict ourselves to the above factors. The self-diffusivity has been obtained from the slope of $u^{2}(\tau)$, mean-squared displacement curves obtained from the MC simulations. Typical plots of $u^{2}(\tau)$ curves are shown in Fig. 5. Due to the reasonably long simulations (60,000 MC steps) a straight line fit to the $u^{2}(\tau)$ curves was found to be quite good, leading to a fairly accurate estimate of $D$.

\section{A. Dependence of $D$ on $\epsilon_{s s}$ and hop length, $h$}

The simplest lattice consists of a uniform surface without any adsorption(lower energy) sites. Figure 6 shows the variation of $D$ vs $c$ for this lattice. The curve shown in Fig. 6(a) decreases monotonically as expected. Kärger et al. ${ }^{17}$ classify this as type I behavior (see Fig. 1). In order to see the effect of the nearest-neighbor interaction, the simulations on the plain lattice were carried out under two differing conditions: (i) $\epsilon_{s s}=-0.5$ and (ii) $\epsilon_{s s}=+20.0 \mathrm{~kJ} / \mathrm{mol}$. The trend for the latter is still a monotonic decrease as shown in Fig. $6 \mathrm{~b}$ but the decrease with $c$ is now more rapid. As expected, the value of $D$ is always lower than the corresponding values obtained for $\epsilon_{s s}=-0.5 \mathrm{~kJ} / \mathrm{mol}$. In order to see the effect of the hop length, simulations were repeated for both $\epsilon_{s s}=$ $-0.5 \mathrm{~kJ} / \mathrm{mol}$ and $\epsilon_{s s}=+20.0 \mathrm{~kJ} / \mathrm{mol}$ with double hops $(h$ $=2)$. It is seen that for both cases, viz., $\epsilon_{s s}=-0.5 \mathrm{~kJ} / \mathrm{mol}$ and $\epsilon_{s s}=+20.0 \mathrm{~kJ} / \mathrm{mol}$, the value of $D$ is significantly higher than for single hops. It is worthwhile noting that for $\epsilon_{s s}=$ $+20.0 \mathrm{~kJ} / \mathrm{mol}$, the $D$ vs $c$ curves deviate significantly from the straight line behavior and possess a distinct positive curvature (second derivative). More important, however, is the fact that all the four $D$ vs $c$ curves are of type I, showing a monotonic decrease with $c$. Note that for values of $c$ above

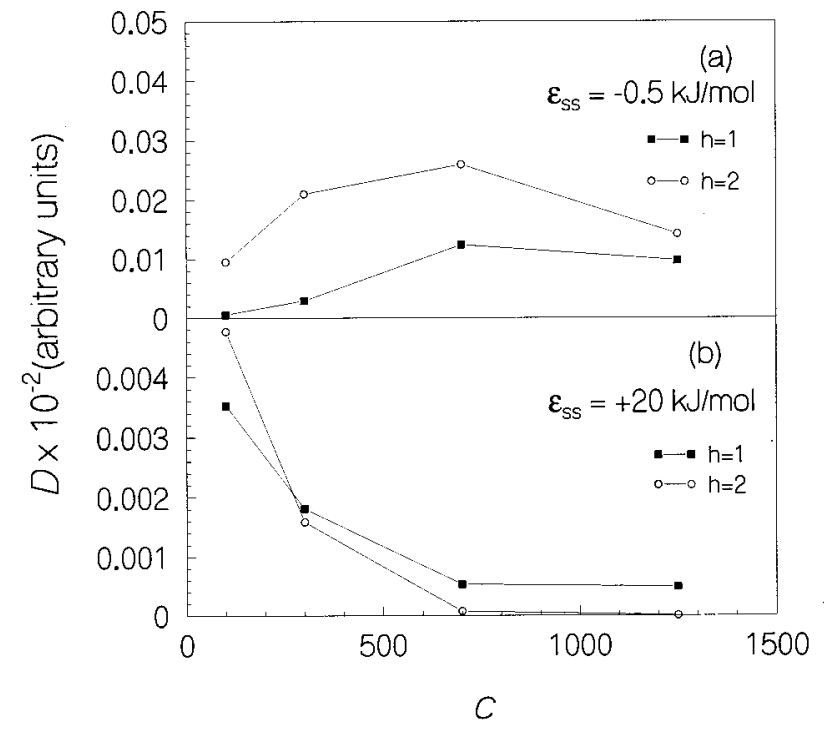

FIG. 7. Results for lattice with square arrangement of adsorption sites [see Fig. 2(b)]. Dependence of self-diffusivity $D$ on sorbate concentration $c$ for (a) $\epsilon_{s s}=-0.5 \mathrm{~kJ} / \mathrm{mol}$ with $h=1$ and 2 . (b) $\epsilon_{s s}=+20 \mathrm{~kJ} / \mathrm{mol}$ with $h=1$ and 2 .

$1250, D$ is expected to decrease with increase in $c$ due to increasing difficulty in finding unoccupied sites, as double occupancy of sites is prohibited.

\section{B. Dependence on adsorption site pattern}

Figures 7 and 8 show the variation of $D$ with $c$ for lattices with adsorption sites for (i) $\epsilon_{s s}=-0.5 \mathrm{~kJ} / \mathrm{mol}$ and $\epsilon_{s s}$ $=20.0 \mathrm{~kJ} / \mathrm{mol}$, and (ii) $h=1$ and $h=2$. Results for two different arrangements of adsorption sites which we term as (i)

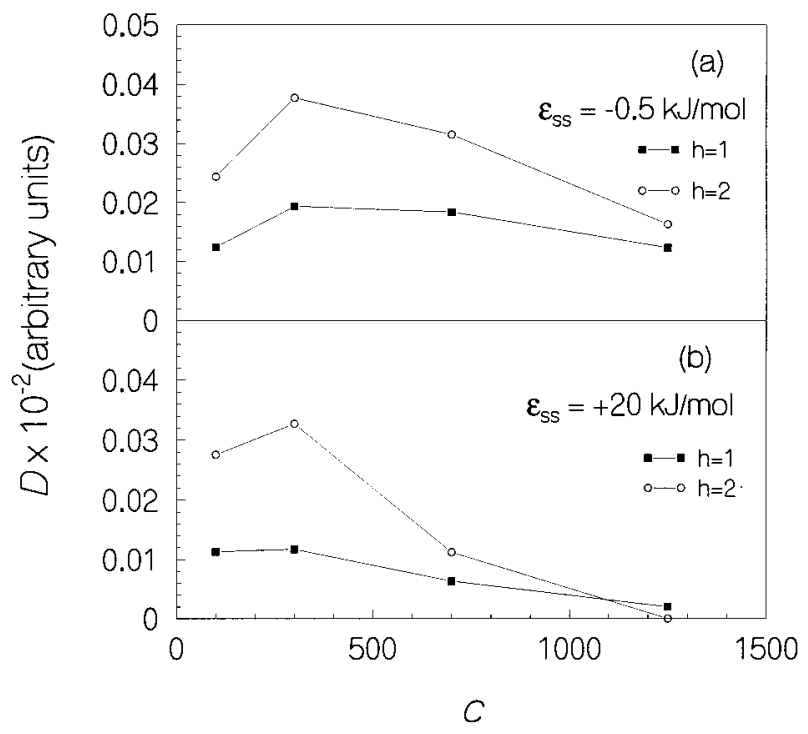

FIG. 8. Results for lattice with clustered pattern of adsorption sites [see Fig. 2(c)]. Dependence of self-diffusivity $D$ on sorbate concentration $c$ for (a) $\epsilon_{s s}=-0.5 \mathrm{~kJ} / \mathrm{mol}$ with $h=1$ and 2. (b) $\epsilon_{s s}=+20 \mathrm{~kJ} / \mathrm{mol}$ with $h=1$ and 2 . 


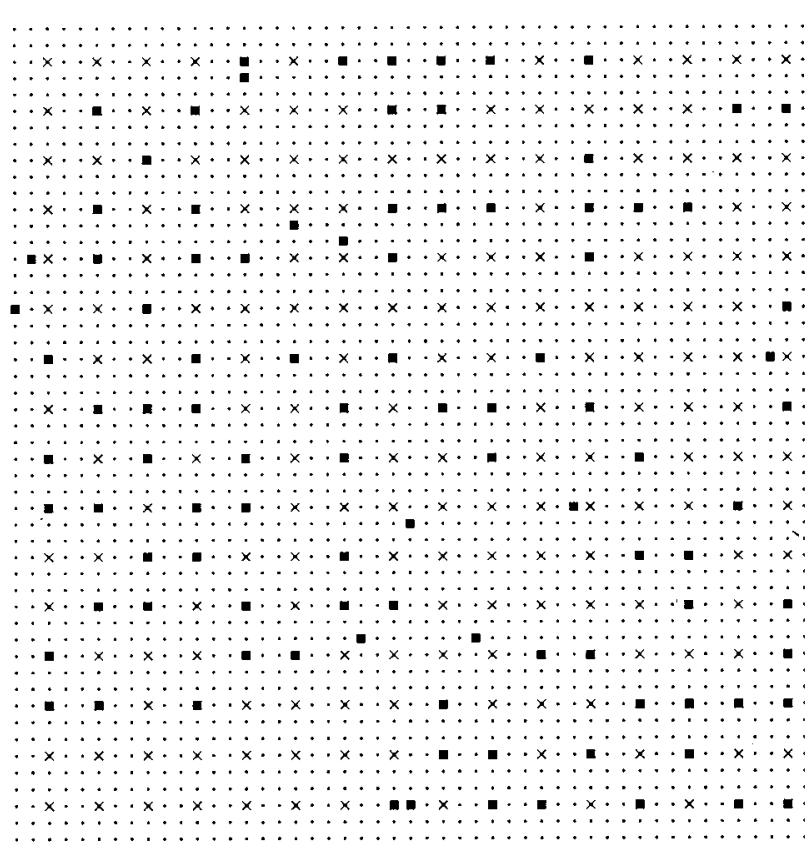

(a)

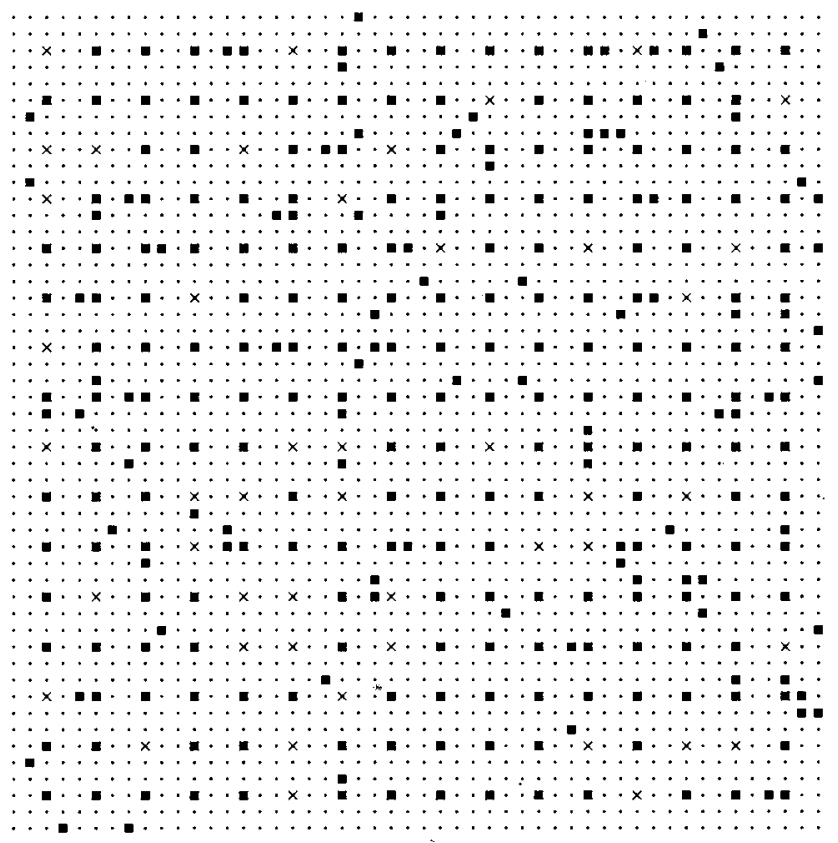

(b)

square and (ii) clustered (see Fig. 2) are shown in Figs. 7 and 8. It is clear from these figures that the simple type I behavior observed for the plain lattice without any adsorption sites goes over to type IV behavior when $\epsilon_{s s}=-0.5 \mathrm{~kJ} / \mathrm{mol}$. The values of the diffusivity are lower in the presence of adsorption sites as a sizable fraction of sorbates is now occupying these sites. This is also responsible for the observed type IV behavior. This is evident from the snapshot pictures of the sorbate positions on the $50 \times 50$ lattice shown in Figs. 9(a), 9(b), and 9(c). The location of the adsorbates for three different concentrations, viz., 100, 300, and 700 sorbates, is indicated on a square arranged lattice. Normal sites and adsorption sites are indicated by dots and crosses, respectively. The filled squares indicate the locations of the adsorbates. It is seen that a large fraction of adsorbates resides on the ad-

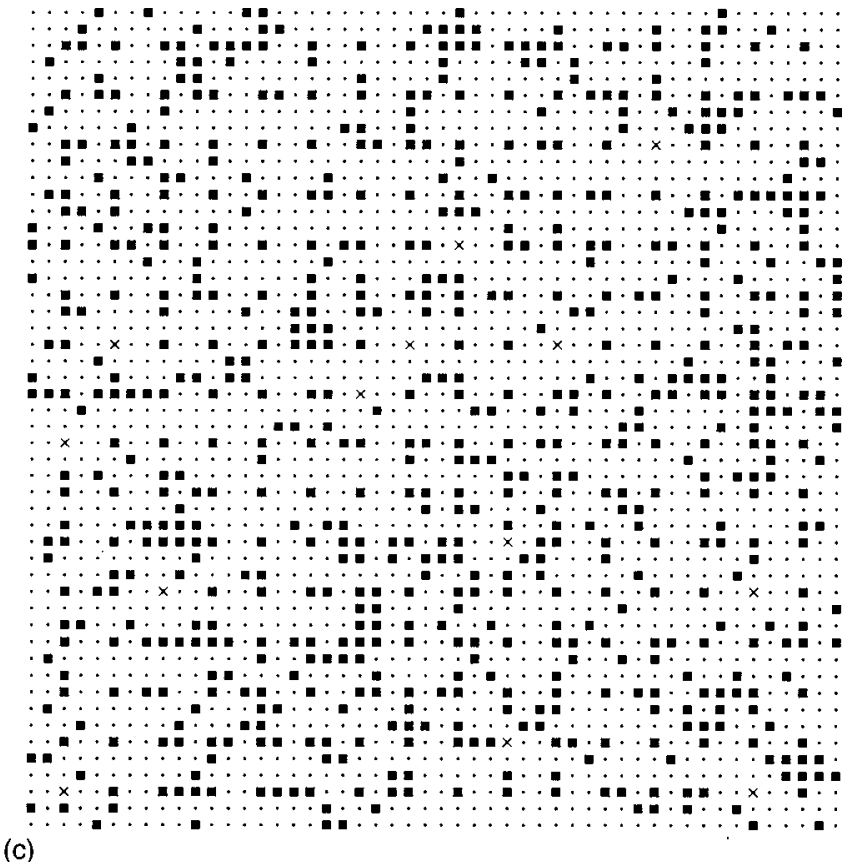

(c)

FIG. 9. Snapshot pictures of $50 \times 50$ lattice with square arrangement of adsorption sites. Here, normal and adsorption sites are indicated by dots and crosses, respectively. The adsorbates are indicated by filled squares. Note that the fraction of sorbates on the normal sites increases steadily with increase in sorbate concentration: $0.11,0.30$, and 0.34 at (a) 100, (b) 300, and (c) 700 sorbates on the lattice consisting of 256 adsorption sites. This results in an increase in self-diffusivity with concentration as observed in Figs. 7 and 8 for square and clustered arrangement of adsorption sites, respectively.

sorption sites: $0.89,0.70$, and 0.66 for concentrations of 100 , 300, and 700 adsorbates, respectively. As can be seen, with increase in adsorbate concentration, a larger fraction of adsorbates occupies normal sites, leading to an increase in the diffusivity.

For $\epsilon_{s s}=+20.0 \mathrm{~kJ} / \mathrm{mol}$, the behavior exhibited is found to be type I or type IV, or even type II depending on the condition under which the simulations are carried out. For the clustered arrangement of sites with $\epsilon_{s s}=+20.0 \mathrm{~kJ} / \mathrm{mol}$, the curve is of type IV when $h=2$ but of type II when $h=1$.

It is clear from this that the nature and strength of sorbate-sorbate interaction, the presence of adsorption sites, and hop length affects the nature of $D$ vs $c$ curves. We can conclude from these results that the presence of adsorption 


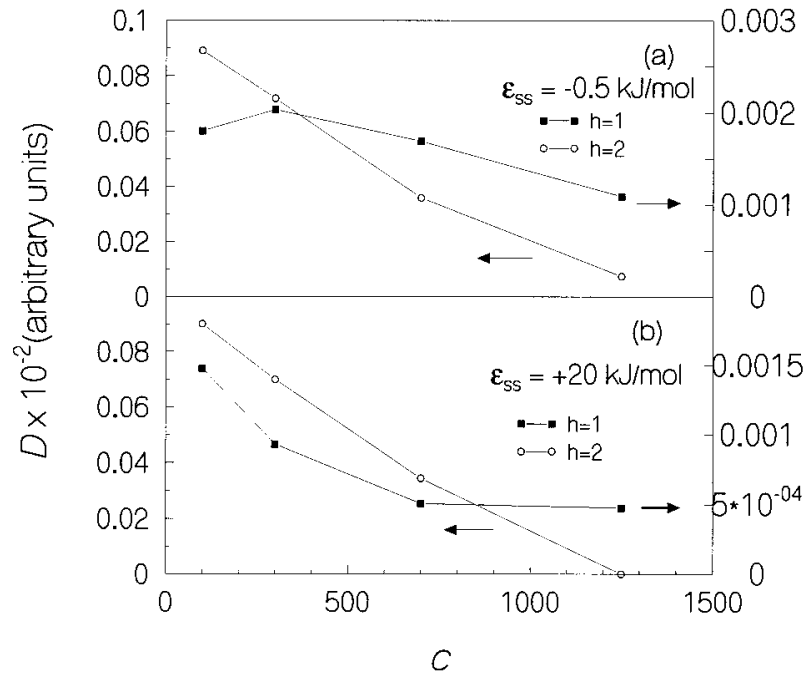

FIG. 10. Results for lattice with chessboard arrangement of adsorption sites [see Fig. 2(d)]. Dependence of self-diffusivity $D$ on sorbate concentration $c$ for (a) $\epsilon_{s s}=-0.5 \mathrm{~kJ} / \mathrm{mol}$ with $h=1$ and 2 . (b) $\epsilon_{s s}=+20 \mathrm{~kJ} / \mathrm{mol}$ with $h=1$ and 2 .

sites makes type I go over to type IV under certain conditions. The nature and strength of sorbate-sorbate interaction can also alter the $D$ vs $c$ behavior so as to give rise to type I or type IV depending on the condition imposed. The hop length can change a type IV to type II behavior under certain conditions $\left(\epsilon_{s s}=+20.0 \mathrm{~kJ} / \mathrm{mol}\right.$ and clustered site distribution). These results suggest that an interplay between different factors can lead to several types of $D$ vs $c$ behavior.

Results for another pattern of adsorption sites-the chessboard pattern-are presented in Fig. 10. The chess- board pattern leads to a type IV behavior similar to that found for square and clustered lattice for the case of $h=1$ (and $\epsilon_{s s}=-0.5 \mathrm{~kJ} / \mathrm{mol}$ ) while the other curves are reminiscent of the plain lattice without adsorption sites. This is because the diffusing sorbates cannot altogether avoid the normal sites for $h=1$; in the case of $h=2$ sorbates can hop from one adsorption site to another without ever occupying a normal site. This is evident in Fig. 11, where open circles and squares represent the normal and adsorption sites, respectively. Filled squares represent the sites occupied by a single adsorbate during its peregrination over about $2000 \mathrm{MC}$ steps. The adsorbate essentially hops from one adsorption site to the next most of the time. In fact, out of these $2000 \mathrm{MC}$ steps (shown in Fig. 11), the adsorbate is found to occupy a normal site only for about five steps. As a consequence, when $h=2$, the whole lattice can be considered similar to a plain lattice. As we saw earlier, the presence of adsorption sites leads to a change of behavior from type I to IV and this is what is observed for $h=1$ and $\epsilon_{s s}=-0.5 \mathrm{~kJ} / \mathrm{mol}$. When $\epsilon_{s s}=+20.0 \mathrm{~kJ} / \mathrm{mol}$ and $h=1$, the repulsion between sorbates prevents occupation of neighboring sites. Besides, all sorbates can occupy the adsorption sites as there is a large number of adsorption sites (1250) leading to very low value for diffusivity (see Fig. 10). When $\epsilon_{s s}=+20.0 \mathrm{~kJ} / \mathrm{mol}$ and $h=2$, sorbates diffuse largely through hops from one adsorption site to a neighboring adsorption site, leading to a high value of $D$.

Calculations have been carried out on clustered arrangement of adsorption sites with $\epsilon_{s s}=-10 \mathrm{~kJ} / \mathrm{mol}$ instead of $\epsilon_{s s}=-0.5 \mathrm{~kJ} / \mathrm{mol}$. The result for $h=2$ is shown in Fig. 12. It is seen that type I behavior is observed. Comparison with the result for clustered arrangement of sites [see Fig. 8(a)]

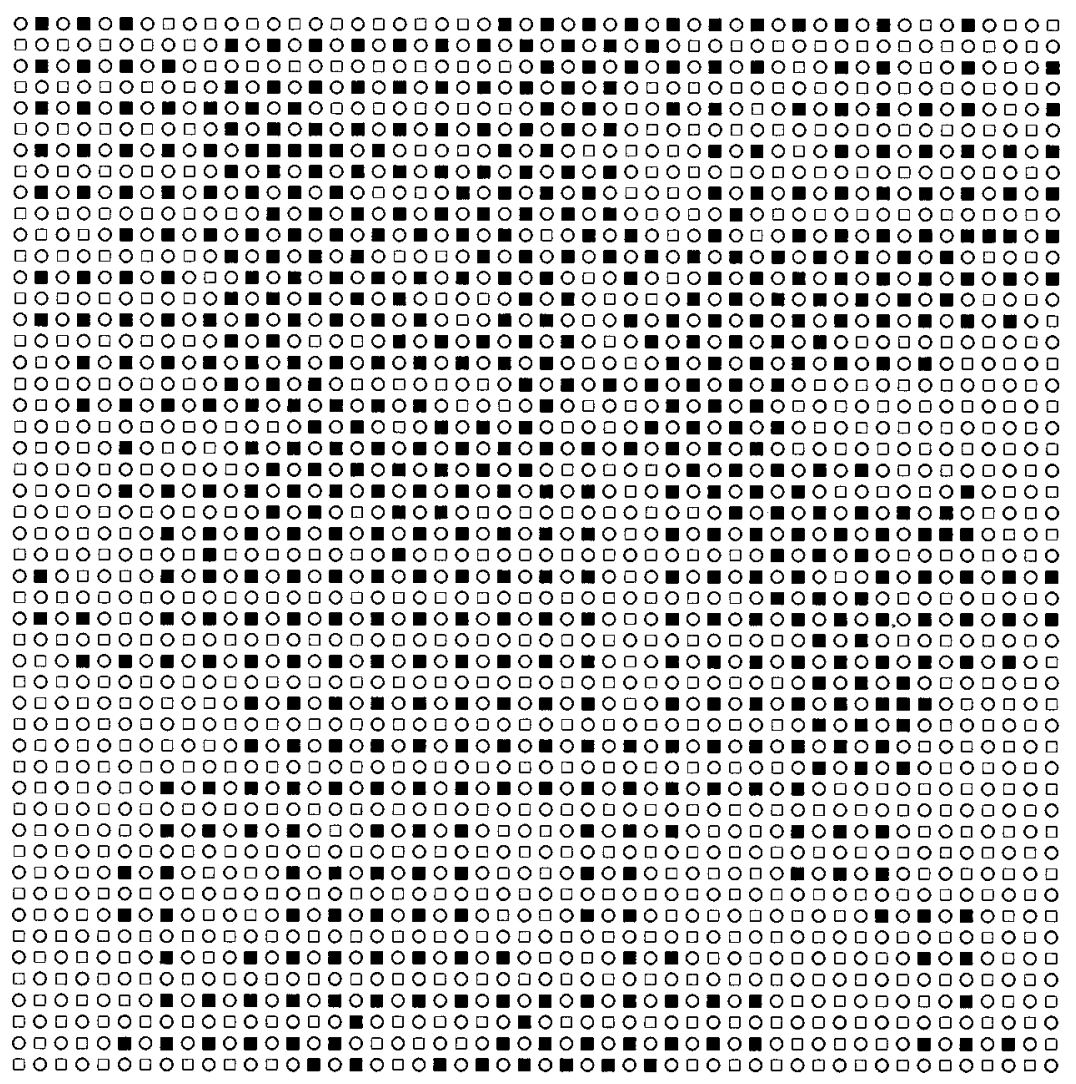

FIG. 11. A trajectory of a single sorbate over 2000 MC steps is shown on a $50 \times 50$ lattice. Normal and adsorption sites are indicated by an open circle and an open square, respectively. The sorbate is indicated by a filled square. The sorbate most of the time hops from one adsorption site to another, avoiding normal sites. This leads to a decrease in $D$ with $c$ similar to that observed in case of a plain lattice. There seems to be only five instances of the sorbate occupying a normal site. 


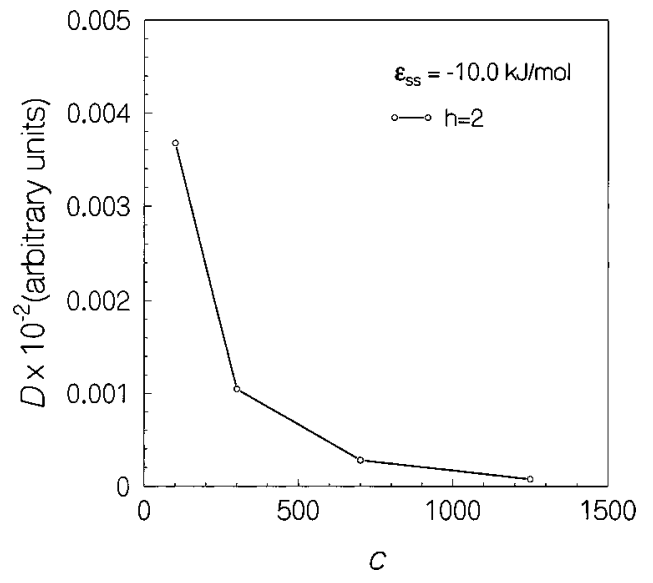

FIG. 12. Effect of change in $\epsilon_{s s}$ on $D$ vs $c$ behavior for clustered arrangement of adsorption sites with $\epsilon_{s s}=-10 \mathrm{~kJ} / \mathrm{mol}$ and $h=2$. Note that type IV behavior observed in Fig. 8(a) for $\boldsymbol{\epsilon}_{s s}=-0.5 \mathrm{~kJ} / \mathrm{mol}$ has changed to type I behavior for $\epsilon_{s s}=-10 \mathrm{~kJ} / \mathrm{mol}$.

with $\epsilon_{s s}=-0.5 \mathrm{~kJ} / \mathrm{mol}, h=2$ suggests that type IV has changed to type I. It is seen that diffusivity is significantly lowered and it decreases much faster with increase in $c$. Stronger sorbate-sorbate interaction leads to clustering of sorbates. Mobility of these clusters is expected to be lower than that of a single sorbate or smaller clusters. At higher concentrations larger clusters are formed and there are fewer clusters of small size, leading to a rapid decrease in $D$ with increase in $c$.

\section{Effect of Confinement}

In order to see the effect of confinement, simulations with "medium" confinement, in which there are windows extending over six lattice sites and "strong" confinement, in which windows extend over just one lattice site, have been carried out (see Fig. 4). The arrangement of adsorption sites is that of clustered arrangement depicted in Fig. 2(c). The results with 6-site and 1-site windows are shown in Figs. 13 and 14, respectively. Figure 13 shows that the dependence of $D$ on $c$ is essentially unchanged when the medium confinement with 6-site windows is introduced. For a 1-site window, there is an overall decrease in the magnitude of $D$. However, for 1-site window and $\epsilon_{s s}=+20 \mathrm{~kJ} / \mathrm{mol}$ and $h=2$, the $D$ vs $c$ curve seems to have changed from type IV to type I (see Fig. 14). But, the difference in values of $D$ between $c=100$ and 300 sorbates is rather small and hence the trend in $D$ vs $c$ is not entirely unambiguous.

\section{CONCLUSIONS}

A summary of different $D$ vs $c$ types obtained in the present study is tabulated in Table I. The different types of behavior of $D$ vs $c$ under different conditions can be understood in terms of the microscopic factors responsible for these. The simple decrease (type I behavior) in $D$ with $c$, observed for a plain lattice is attributed to decrease in the mean free path of the sorbates as sorbate concentration is increased. This is the behavior observed for a bulk gas as well and is to be expected. Introduction of adsorption sites with stronger attraction, i.e., lower on-site energy, leads to

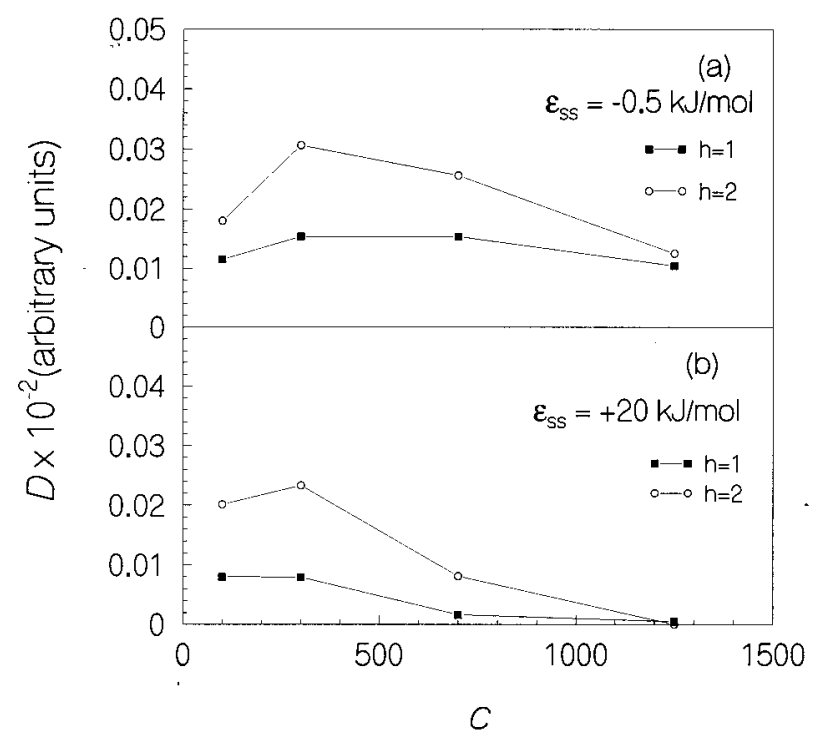

FIG. 13. Dependence of $D$ on $c$ for lattice with medium confinement (6-site window) and clustered arrangement of sites for (a) $\epsilon_{s s}=-0.5 \mathrm{~kJ} / \mathrm{mol}$ and $h=1$ and 2 . (b) $\epsilon_{s s}=+20 \mathrm{~kJ} / \mathrm{mol}$ and $h=1$ and 2 .

type I behavior going over to type IV behavior. At low sorbate concentrations, most of the adsorbates are bound to the adsorption sites, leading to a low value for $D$. With increase in the number of sorbates, due to the limited availability of adsorption sites, there is an increase in the fraction of sorbates that is not bound to the adsorption sites. This contributes to an increase in the value of $D$ with $c$. At still higher values of $c$, mean free path decreases, which causes a decrease in $D$.

The case of the chessboard pattern is similar to that of plain lattice when $h=2$. This leads to type I behavior. This is because sorbates can entirely avoid occupying a normal site when $h=2$. When $h=1$, the expected $D$ vs $c$ behavior is

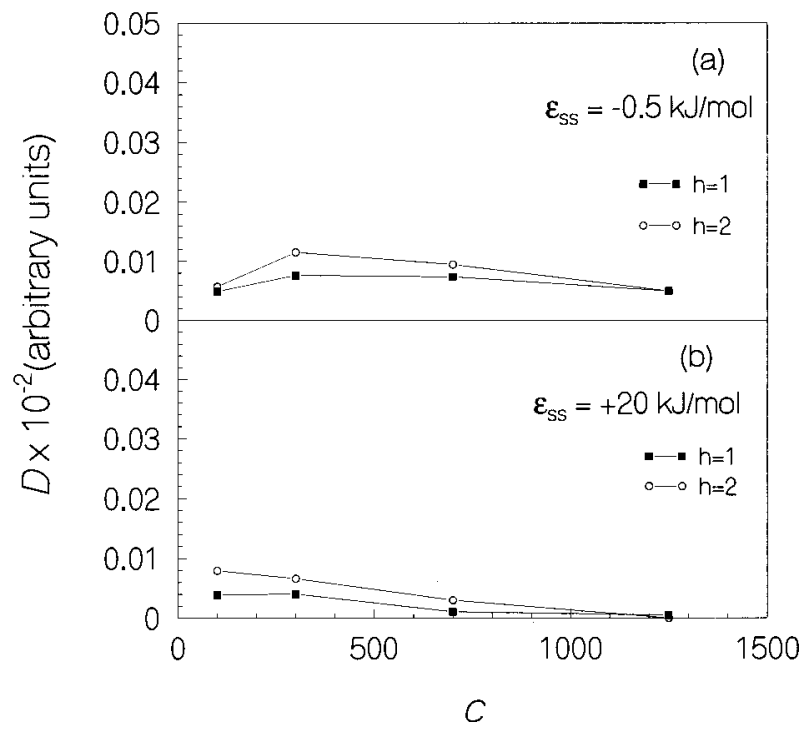

FIG. 14. Dependence of $D$ on $c$ for lattice with strong confinement (1-site window) and clustered arrangement of sites for (a) $\epsilon_{s s}=-0.5 \mathrm{~kJ} / \mathrm{mol}$ and $h=1$ and 2 . (b) $\epsilon_{s s}=+20 \mathrm{~kJ} / \mathrm{mol}$ and $h=1$ and 2 . 
TABLE I. Summary of results on dependence of self-diffusivity on sorbate concentration from 2-dimensional lattice gas simulations. Different types of $D$ vs $c$ curves are as depicted in Fig. 1 (Ref. 17).

\begin{tabular}{|c|c|c|c|c|}
\hline \multirow[b]{2}{*}{ Pattern of adsorption sites } & \multicolumn{2}{|c|}{$\epsilon_{s s}=-0.5 \mathrm{~kJ} / \mathrm{mol}$} & \multicolumn{2}{|c|}{$\epsilon_{s s}=+20.0 \mathrm{~kJ} / \mathrm{mol}$} \\
\hline & $h=1$ & $h=2$ & $h=1$ & $h=2$ \\
\hline None & I & I & I & I \\
\hline Square arranged & IV & IV & I & I \\
\hline Chess board & IV & I & I & I \\
\hline \multicolumn{5}{|l|}{ Clustered } \\
\hline (i) no confinement & IV & IV & $\mathrm{II} / \mathrm{I}$ & IV \\
\hline (ii) confinement with 6-site window & IV & IV & II & IV \\
\hline (iii) confinement with 1-site window & IV & IV & $\mathrm{II} / \mathrm{I}$ & I \\
\hline
\end{tabular}

similar to a lattice with adsorption sites. Therefore, one finds a type IV dependence. When $h=1$, a sorbate has to occupy a normal site while diffusing on the lattice. It is therefore understandable that the observed $D$ vs $c$ curve is of the type IV for $h=1$ and of type I for $h=2$.

The effect of stronger sorbate-sorbate interaction on lattices with clustered adsorption sites is to lower the diffusivity, and the $D$ vs $c$ type IV dependence goes over to type I. This is because of a significant increase in the formation of clusters with increase in the sorbate concentration, $c$, even at low $c$. The decrease in $D$ with $c$ is steeper. The clusters increase rapidly both in size and number with increase in $c$, leading to a more rapid decrease in $D$ with $c$.

Confinement as introduced in the present work leads to type IV behavior going over to type I for a 1-site window with $h=2$. More detailed studies are required to explore the effect of confinement under different conditions.

In conclusion, at least three different types of $D$ vs $c$ behavior, viz., types I, II, and IV, have been obtained in the present study. It is found that introduction of adsorption sites is responsible for the type $\mathrm{I} \rightarrow \mathrm{IV}$ transition. A type IV to I transition can be obtained either by removal of adsorption sites or by an increase in the strength of adsorbate-adsorbate interactions or by the introduction of strong confinement. A tuning of the adsorption sites with the adsorbate-adsorbate interactions can lead to type II behavior.

In relation to the question of self-diffusivities obtained from Metropolis Monte Carlo and other allied methods, we found, after this work was completed, the work by Huitema and van der Eerden. They have addressed the question of the relationship between a Monte Carlo and a molecular dynamics step and compared the self-diffusivities obtained from the two methods. ${ }^{38}$

\section{ACKNOWLEDGMENTS}

The authors wish to acknowledge the support of Council of Scientific and Industrial Research, New Delhi for partial financial support of this research through a grant and also award of Junior Research Fellowship to one of us (S.Y.B.). Partial financial support from the Department of Science and Technology is also acknowledged. Partial financial support from the Jawaharlal Nehru Center for Advanced Scientific Research is gratefully acknowledged. Computational re- sources from Supercomputer Education and Research Center are acknowledged. Contribution No. 1346 from the Solid State and Structural Chemistry Unit.

${ }^{1}$ P. Demontis and G. B. Suffritti, Chem. Rev. 97, 2845 (1997)

${ }^{2}$ S. P. Bates and R. A. van Santen, Adv. Catal. 42, 1 (1998).

${ }^{3}$ H. Klein, H. Fuess, and G. Schrimpf, J. Phys. Chem. 100, 11101 (1996).

${ }^{4}$ M. Miyahara and K. E. Gubbins, J. Chem. Phys. 106, 2865 (1997).

${ }^{5}$ T. Mosell, G. Schrimpf, and J. Brickmann, J. Phys. Chem. 100, 4582 (1996)

${ }^{6}$ P. Demontis, G. B. Suffritti, and A. Tilocca, J. Chem. Phys. 105, 5586 (1996).

${ }^{7}$ F. Vigné-Maeder, J. Phys. Chem. 98, 4666 (1994).

${ }^{8}$ E. Hernandez and C. R. A. Catlow, Proc. R. Soc. London, Ser. A 448, 143 (1995).

${ }^{9}$ S. H. Lee, G. K. Moon, S. G. Choi, and H. S. Kim, J. Phys. Chem. 98, 1561 (1994).

${ }^{10}$ R. L. June, A. T. Bell, and D. N. Theodorou, J. Phys. Chem. 94, 8232 (1990).

${ }^{11}$ E. G. Derouane, J. M. Andre, L. Leherte, P. J. Galet, D. Vanderveken, D. P. Vercauteren and G. Fripiat, in Theoretical Aspects of Heterogeneous Catalysis, edited by J. B. Moffat (Van Nostrand Reinhold, New York, 1990).

${ }^{12}$ L. M. Bull, N. J. Hensen, A. K. Cheetham, J. M. Newsam, and S. J. Heyes, J. Phys. Chem. 97, 11776 (1993).

${ }^{13}$ R. M. Barrer, Zeolites and Clay Minerals as Sorbents and Molecular Sieves (Academic, New York, 1978) pp. 288-289.

${ }^{14}$ P. R. van Tassel, H. T. Davis, and A. V. McCormick, Langmuir 12, 1257 (1994); K. G. Ayappa, ibid. 14, 880 (1998).

${ }^{15}$ K. Hahn, J. Kärger, and V. Kukla, Phys. Rev. Lett. 76, 2762 (1996).

${ }^{16}$ S. Yashonath and P. Santikary, J. Phys. Chem. 98, 6368 (1994).

${ }^{17}$ J. Kärger and H. Pfeifer, Zeolites 7, 90 (1987).

${ }^{18}$ C. Uebing, V. Pereyra, and G. Zgrablich, Surf. Sci. 366, 185 (1996).

${ }^{19}$ C. Uebing, V. Pereyra, and G. Zgrablich, J. Chem. Phys. 106, 9320 (1997).

${ }^{20}$ C. Rödenbeck, J. Kärger, and K. Hahn, Phys. Rev. E 55, 5697 (1997).

${ }^{21}$ F. Jousse, L. Leherte, and D. P. Vercauteren, J. Phys. Chem. B 101, 4717 (1997).

${ }^{22}$ C. Saravanan, F. Jousse, and S. M. Auerbach, Phys. Rev. Lett. 80, 5754 (1998).

${ }^{23}$ Z. Zhang, K. Haug, and H. Metiu, J. Chem. Phys. 93, 3614 (1990).

${ }^{24}$ R. L. June, A. T. Bell, and D. N. Theodorou, J. Phys. Chem. 95, 8866 (1991)

${ }^{25}$ S. M. Auerbach, N. J. Hensen, A. K. Cheetham, and H. I. Metiu, J. Phys. Chem. 99, 10600 (1995).

${ }^{26}$ S. M. Auerbach and H. I. Metiu, J. Chem. Phys. 106, 2893 (1997).

${ }^{27}$ S. M. Auerbach, J. Chem. Phys. 106, 7810 (1997).

${ }^{28} \mathrm{~K}$. W. Kehr and K. Binder, in Applications of the Monte Carlo Method in Statistical Physics, 2nd ed., edited by K. Binder (Springer, Berlin, 1987).

${ }^{29}$ K. A. Fichthorn and W. H. Weinberg, J. Chem. Phys. 95, 1090 (1991).

${ }^{30}$ H. C. Kang and W. H. Weinberg, J. Chem. Phys. 90, 2824 (1989). 
${ }^{31}$ H. C. Kang and W. H. Weinberg, Acc. Chem. Res. 25, 253 (1992).

${ }^{32}$ P. Santikary, S. Yashonath, and G. Ananthakrishna, J. Phys. Chem. 96, 10469 (1992).

${ }^{33}$ W. L. Jorgensen, J. D. Madura, and C. J. Swensen, J. Am. Chem. Soc. 106, 6638 (1984).

${ }^{34}$ S. J. Goodbody, K. Watanabe, D. MacGowan, J. P. R. B. Walton, and N. Quirke, J. Chem. Soc., Faraday Trans. 87, 1951 (1991).
${ }^{35}$ R. M. Barrer and W. Jost, Trans. Faraday Soc. 45, 928 (1949).

${ }^{36}$ M. Azzouz, H. J. Kreuzer, and M. R. A. Shegelski, Phys. Rev. Lett. 80, 1477 (1998).

${ }^{37}$ N. Metropolis, A. W. Rosenbluth, M. N. Rosenbluth, A. H. Teller, and E. Teller, J. Chem. Phys. 21, 1087 (1953).

${ }^{38}$ H. E. A. Huitema and J. P. van der Eerden, J. Chem. Phys. 110, 3267 (1999). 